\title{
ON THE DYNAMICS OF THE EULER EQUATIONS $\mathrm{ON} \mathrm{SO}(4)$
}

\author{
CLAUDIO A. BUZZI ${ }^{1}$ JAUME LLIBRE ${ }^{2}$ AND RUBENS PAZIM ${ }^{3}$
}

\begin{abstract}
This paper deals with the Euler equations on the Lie Algebra $s o(4)$. These equations are given by a polynomial differential system in $\mathbb{R}^{6}$. We prove that this differential system has four 3-dimensional invariant manifolds and we give a complete description of its dynamics on these invariant manifolds. In particular, each of these invariant manifolds are fulfilled by periodic orbits except in a zero Lebesgue measure set.
\end{abstract}

\section{INTRODUCTION}

The Euler equations on the Lie algebra $s o(4)$ have a very long story that can be found in $[1,2,3,5,6,7,8]$ and in the references quoted therein. The Euler differential equations on so(4) in $\mathbb{R}^{6}$ depend on six parameters $\lambda_{i}, i=1, \ldots, 6$, and are given by

$$
\begin{aligned}
& \dot{x_{1}}=\left(\lambda_{3}-\lambda_{2}\right) x_{2} x_{3}+\left(\lambda_{6}-\lambda_{5}\right) x_{6} x_{5}, \\
& \dot{x_{2}}=\left(\lambda_{1}-\lambda_{3}\right) x_{1} x_{3}+\left(\lambda_{4}-\lambda_{6}\right) x_{4} x_{6}, \\
& \dot{x_{3}}=\left(\lambda_{2}-\lambda_{1}\right) x_{1} x_{2}+\left(\lambda_{5}-\lambda_{4}\right) x_{4} x_{5}, \\
& \dot{x_{4}}=\left(\lambda_{3}-\lambda_{5}\right) x_{3} x_{5}+\left(\lambda_{6}-\lambda_{2}\right) x_{2} x_{6}, \\
& \dot{x_{5}}=\left(\lambda_{4}-\lambda_{3}\right) x_{3} x_{4}+\left(\lambda_{1}-\lambda_{6}\right) x_{1} x_{6}, \\
& \dot{x_{6}}=\left(\lambda_{2}-\lambda_{4}\right) x_{2} x_{4}+\left(\lambda_{5}-\lambda_{1}\right) x_{1} x_{5} .
\end{aligned}
$$

They can have a very complicated dynamics, due to the complexity of the system: nonlinear, high dimension and many parameters. From the integrability point of view, in the paper [4] it is proved that the Euler equations on the Lie algebra so(4) with a diagonal quadratic Hamiltonian either satisfy the Manakov condition, or have at most four functionally independent polynomial first integrals.

In the present paper our first interest was about the existence or non-existence of periodic orbits for system (1). As we will see in what follows, we have proved that there exist 3-dimensional invariant manifolds for the flow of system (1) that are fulfilled by periodic orbits.

\footnotetext{
Key words and phrases. Euler equations on so(4); Periodic Orbits; Reversible systems .
} 
In our first theorem we prove the existence of four invariant manifolds and show that the restriction of system (1) to these invariant manifolds is completely integrable. In order to state our result we define the following manifolds:

$$
\begin{aligned}
& W_{1}=\left\{\mathbf{x}: x_{1}=x_{2}=x_{6}=0\right\}, \\
& W_{2}=\left\{\mathbf{x}: x_{1}=x_{3}=x_{5}=0\right\}, \\
& W_{3}=\left\{\mathbf{x}: x_{2}=x_{3}=x_{4}=0\right\}, \\
& W_{4}=\left\{\mathbf{x}: x_{4}=x_{5}=x_{6}=0\right\},
\end{aligned}
$$

where $\mathbf{x}=\left(x_{1}, \ldots, x_{6}\right)$.

Theorem 1. The following statements hold.

(i) System (1) has the four 3-dimensional invariant manifolds $W_{i}$ for $i=1, \ldots, 4$.

(ii) System (1) restricted to each invariant manifold $W_{i}, i=1, \ldots, 4$, is of the form

$$
\dot{x}=(\gamma-\beta) y z, \quad \dot{y}=(\alpha-\gamma) x z, \quad \dot{z}=(\beta-\alpha) x y .
$$

(iii) The functions $F_{1}(x, y, z)=x^{2}+y^{2}+z^{2}$ and $F_{2}(x, y, z)=\alpha x^{2}+$ $\beta y^{2}+\gamma z^{2}$ are first integrals of system (2).

In our second theorem we provide the dynamics on the invariant manifolds $W_{i}$.

Theorem 2. Consider system (2) with $\alpha \neq \beta, \alpha \neq \gamma$ and $\beta \neq \gamma$ (without loss of generality we can assume $\alpha<\beta<\gamma$ ). For each $r>0$ the sphere $S_{r}=\left\{x^{2}+y^{2}+z^{2}=r\right\}$ is an invariant manifold by the flow of system (2), and the dynamics on these sphere is described in Figure 1. More precisely:

(i) The equilibrium points on $S_{r}$ are $(0,0, \pm r)$ (centers), $(0, \pm r, 0)$ (saddles) and $( \pm r, 0,0)$ (centers).

(ii) On the sphere $S_{r}$ there are four heteroclinic connections: two connections from the point $(0, r, 0)$ to the point $(0,-r, 0)$ and two connections from the point $(0,-r, 0)$ to the point $(0, r, 0)$.

(iii) All orbits that are neither equilibrium points, nor heteroclinic connections are periodic.

The paper is organized as follows. In section 2 we present a brief description of the reversible and equivariant differential systems, which are the main tool used in our work. In sections 3 and 4 we give the proofs of Theorem 1 and 2. 


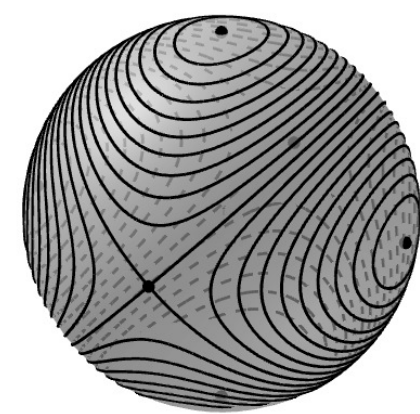

FiguRe 1. Phase space of system (2) with $\alpha<\beta<\gamma$ on the sphere $S_{r}$.

\section{Reversible and Equivariant Systems}

We consider a vector field $f: \mathbb{R}^{6} \rightarrow \mathbb{R}^{6}$, generating a dynamical system, i.e. the flow of

$$
\frac{d \mathbf{x}}{d t}=f(\mathbf{x})
$$

Let $R$ be an involution, i.e., a diffeomorphism of $\mathbb{R}^{6}$ to itself in such a way that $R \circ R=I d$. Then $f$ is called $R$-reversible if $R_{*} f=-f \circ R$, i.e., $d R_{p}(f(p))=-f(R(p))$. So system (3) has a solution $\mathbf{x}(t)$ if and only if $R \mathbf{x}(-t)$ is a solution too. In this case we say that $R$ is a reversing symmetry of the vector field $f$. Analogously, considering $S$ another involution, $X$ is called $S$-equivariant if $S_{*} f=f \circ S$, in this case every solution $\mathbf{x}(t)$ implies the existence of a solution $S \mathbf{x}(t)$, and we say $S$ is a symmetry of $f$.

The sets of fixed points Fix $(R)=\left\{\mathbf{x} \in \mathbb{R}^{6}: R(\mathbf{x})=\mathbf{x}\right\}$ and Fix $(S)=\left\{\mathbf{x} \in \mathbb{R}^{6}: S(\mathbf{x})=\mathbf{x}\right\}$ play important roles. One of these roles is given by the next lemma. The fixed set Fix $(S)$ is invariant under the flow generated by $f$, that is, a trajectory with a point in the fixed set will remain in it for all time.

Lemma 1. If $f$ is an S-equivariant vector field, then Fix $(S)$ is an invariant manifold under the flow of $f$.

Proof. This follows from the fact that $d S_{p}(f(p))=f(S(p))=f(p)$ for all $p \in F i x(S)$.

Another important property is the following. 
Lemma 2. Let $R_{1}$ and $R_{2}$ be two involutions from $\mathbb{R}^{6}$ to $\mathbb{R}^{6}$ such that $S=R_{1} \circ R_{2}$ is also an involution. If the vector field $X$ is reversible with respect to the involutions $R_{1}$ and $R_{2}$, then it is $S$-equivariant.

Proof. It is clear that if $R_{i_{*}} f=-f \circ R_{i}$ for $i=1,2$, then $\left(R_{1} \circ R_{2}\right)_{*} f=$ $f \circ\left(R_{1} \circ R_{2}\right)$.

Next lemma gives additional properties of the reversible systems that will be useful in our work.

Lemma 3. Let $R$ be a reversing symmetry of the vector field $f$.

(i) If $P$ is an equilibrium point of $f$ such that $P \notin F i x(R)$, then $P^{\prime}=R(P) \neq P$ is also an equilibrium point of $f$. In this case, if $\lambda$ is an eigenvalue of $d f_{P}$, then $-\lambda$ is an eigenvalue of $d f_{P^{\prime}}$. In particular, if $P$ is a hyperbolic saddle then $P^{\prime}$ also is a hyperbolic saddle.

(ii) If $\mathbf{x}(t)$ is a solution of system (3) such that $\lim _{t \rightarrow \pm \infty} \mathbf{x}(t)=P$, then the solution $\mathbf{y}(t)=R(\mathbf{x}(-t))$ satisfies $\lim _{t \rightarrow \mp \infty} \mathbf{y}(t)=P^{\prime}$. In particular, if $\mathbf{x}(t)$ is such that $\mathbf{x}(0) \in$ Fix $(R)$, then $\Gamma=$ $\{\mathbf{x}(t): t \in \mathbb{R}\}=\{R(\mathbf{x}(-t)): t \in \mathbb{R}\}$ is a heteroclinic orbit connecting $P$ and $P^{\prime}$.

Proof. It is clear that if $P \notin F i x(R)$, then $P^{\prime}=R(P) \neq P$. On the other hand if $f(P)=0$, then $f\left(P^{\prime}\right)=f(R(P))=-d R_{P}(f(P))=$ $-d R_{P}(0)=0$. Derivating the expression of the reversibility we obtain $d R_{P} \circ d f_{P} \circ\left(d R_{P}\right)^{-1}=-d f_{P^{\prime}}$, so it is clear that if $\lambda$ is an eigenvalue of $d f_{P}$ then $-\lambda$ is an eigenvalue of $d f_{P^{\prime}}$. The first part of statement (ii) follows by the continuity of the involution $R$. And the second part follows by the Existence and Uniqueness Theorem of ODE's, because both solutions $\mathbf{x}(t)$ and $R(\mathbf{x}(-t))$ coincide at $t=0$.

\section{Proof of Theorem 1}

This section is devoted to give the proof of Theorem 1 .

Proof of Theorem 1. According to the previous section it is easy to see that system (1) is $R_{i}$-reversible with respect to the involutions $R_{i}$ : 
$\mathbb{R}^{6} \rightarrow \mathbb{R}^{6}, i=1, \ldots, 8$, given by

$$
\begin{aligned}
& R_{1}(\mathbf{x})=\left(x_{1}, x_{2},-x_{3}, x_{4}, x_{5},-x_{6}\right), \\
& R_{2}(\mathbf{x})=\left(x_{1}, x_{2},-x_{3},-x_{4},-x_{5}, x_{6}\right), \\
& R_{3}(\mathbf{x})=\left(x_{1},-x_{2}, x_{3}, x_{4},-x_{5}, x_{6}\right), \\
& R_{4}(\mathbf{x})=\left(x_{1},-x_{2}, x_{3},-x_{4}, x_{5},-x_{6}\right), \\
& R_{5}(\mathbf{x})=\left(-x_{1}, x_{2}, x_{3}, x_{4},-x_{5},-x_{6}\right), \\
& R_{6}(\mathbf{x})=\left(-x_{1}, x_{2}, x_{3},-x_{4}, x_{5}, x_{6}\right), \\
& R_{7}(\mathbf{x})=\left(-x_{1},-x_{2},-x_{3}, x_{4}, x_{5}, x_{6}\right), \\
& R_{8}(\mathbf{x})=\left(-x_{1},-x_{2},-x_{3},-x_{4},-x_{5},-x_{6}\right) .
\end{aligned}
$$

Now for $i, j=1, \ldots, 8$ we use the notation $S_{i j}$ to denote the composition $S_{i j}=R_{i} \circ R_{j}$. So, by Lemma 2, we have that system (1) is $S_{i j}$-equivariant with respect to these involutions:

$$
\begin{aligned}
& S_{18}(\mathbf{x})=S_{27}(\mathbf{x})=S_{36}(\mathbf{x})=S_{45}(\mathbf{x})=\left(-x_{1},-x_{2}, x_{3},-x_{4},-x_{5}, x_{6}\right), \\
& S_{16}(\mathbf{x})=S_{25}(\mathbf{x})=S_{38}(\mathbf{x})=S_{47}(\mathbf{x})=\left(-x_{1}, x_{2},-x_{3},-x_{4}, x_{5},-x_{6}\right), \\
& S_{13}(\mathbf{x})=S_{24}(\mathbf{x})=S_{57}(\mathbf{x})=S_{68}(\mathbf{x})=\left(x_{1},-x_{2},-x_{3}, x_{4},-x_{5},-x_{6}\right), \\
& S_{17}(\mathbf{x})=S_{28}(\mathbf{x})=S_{35}(\mathbf{x})=S_{46}(\mathbf{x})=\left(-x_{1},-x_{2}, x_{3}, x_{4}, x_{5},-x_{6}\right), \\
& S_{15}(\mathbf{x})=S_{26}(\mathbf{x})=S_{37}(\mathbf{x})=S_{48}(\mathbf{x})=\left(-x_{1}, x_{2},-x_{3}, x_{4},-x_{5}, x_{6}\right), \\
& S_{14}(\mathbf{x})=S_{23}(\mathbf{x})=S_{58}(\mathbf{x})=S_{67}(\mathbf{x})=\left(x_{1},-x_{2},-x_{3},-x_{4}, x_{5}, x_{6}\right), \\
& S_{12}(\mathbf{x})=S_{34}(\mathbf{x})=S_{56}(\mathbf{x})=S_{78}(\mathbf{x})=\left(x_{1}, x_{2}, x_{3},-x_{4},-x_{5},-x_{6}\right) .
\end{aligned}
$$

From Lemma 1 we obtain the following 3-dimensional invariant manifolds

$$
\begin{aligned}
& W_{1}=F i x\left(S_{17}\right)=\left\{\mathbf{x}: x_{1}=x_{2}=x_{6}=0\right\}, \\
& W_{2}=F i x\left(S_{15}\right)=\left\{\mathbf{x}: x_{1}=x_{3}=x_{5}=0\right\}, \\
& W_{3}=\operatorname{Fix}\left(S_{14}\right)=\left\{\mathbf{x}: x_{2}=x_{3}=x_{4}=0\right\}, \\
& W_{4}=\operatorname{Fix}\left(S_{12}\right)=\left\{\mathbf{x}: x_{4}=x_{5}=x_{6}=0\right\},
\end{aligned}
$$

for system (1).

The proof of statement $(i i)$ is very similar for each one of the invariant manifolds $W_{i}$. We prove it only for the invariant manifold $W_{1}$. System (1) restrict to $W_{1}$ is given by

(4) $\dot{x_{3}}=\left(\lambda_{5}-\lambda_{4}\right) x_{4} x_{5}, \quad \dot{x_{4}}=\left(\lambda_{3}-\lambda_{5}\right) x_{3} x_{5}, \quad \dot{x_{5}}=\left(\lambda_{4}-\lambda_{3}\right) x_{3} x_{4}$,

and obviously it is a system (2).

Finally the proof of statement (iii), for system (2) follows from the fact that

$$
\frac{\partial F_{i}}{\partial x} \dot{x}+\frac{\partial F_{i}}{\partial y} \dot{y}+\frac{\partial F_{i}}{\partial z} \dot{z}=0,
$$

for all $(x, y, z) \in \mathbb{R}^{3}$ and $i=1,2$. 


\section{Proof of Theorem 2}

Proof of Theorem 2. Since $F_{1}(x, y, z)=x^{2}+y^{2}+z^{2}$ is a first integral of system (2), then the sphere $S_{r}$ is an invariant manifold for this system. In what follows we will prove statements (i), (ii) and (iii).

For statement (i) it is easy to show that $(0,0, \pm r),(0, \pm r, 0)$ and $( \pm r, 0,0)$ are equilibrium point of system $(2)$. Now we need to analyze the dynamics of the system in a neighborhood of these equilibrium points. For this purpose consider the parametrizations $z=$ $\pm \sqrt{r-x^{2}-y^{2}}, y= \pm \sqrt{r-x^{2}-z^{2}}$ and $x= \pm \sqrt{r-y^{2}-z^{2}}$ to understand the dynamics in the hemispheres $z>0(z<0), y>0(y<0)$ and $x>0(x<0)$ respectively. We started with $z=\sqrt{r-x^{2}-y^{2}}$. Then system (2) restricted to the surface $\left\{x^{2}+y^{2}+z^{2}=r, z>0\right\}$ can be written as

$$
\dot{x}=(\gamma-\beta) y \sqrt{r-x^{2}-y^{2}}, \quad \dot{y}=(\alpha-\gamma) x \sqrt{r-x^{2}-y^{2}} .
$$

Rescaling the independent variable of the previous differential system in a neighborhood of the equilibrium point $(0,0, r)$ its dynamics is equivalent to the one of the following system

$$
\dot{x}=(\gamma-\beta) y, \quad \dot{y}=(\alpha-\gamma) x .
$$

As system (5) is linear and $\alpha<\beta<\gamma$, then the point $(0,0, r)$ is a linear center, see Figure 2. By symmetry the equilibrium point $(0,0,-r)$ is also a center.

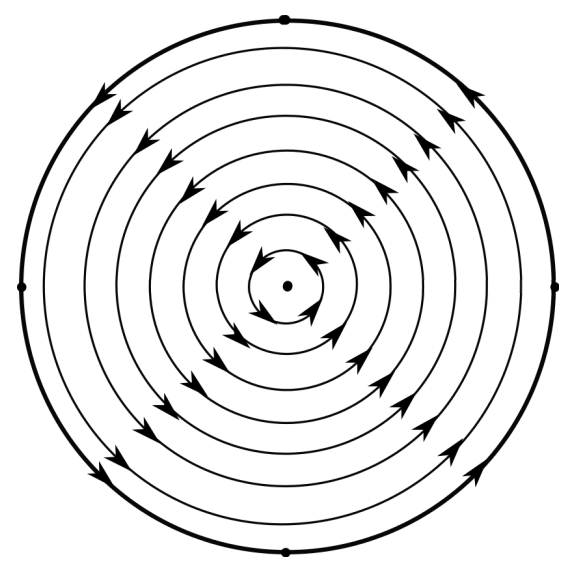

Figure 2. Phase portrait in a neighborhood of the equilibrium point $(r, 0,0)$ of system $(2)$.

Analogously, using a convenient time re-parametrization the dynamics of system (2) restricted to the surface $\left\{x^{2}+y^{2}+z^{2}=r, y>0\right\}$ 
in a neighborhood of the equilibrium point $(0, r, 0)$ is equivalent to the dynamics of the system

$$
\dot{x}=(\gamma-\beta) z, \quad \dot{z}=(\beta-\alpha) x .
$$

Again by the linearity of these system and the hypothesis $\alpha<\beta<\gamma$, the equilibrium point $(0, r, 0)$ is saddle. The symmetry ensures that the equilibrium point $(0,-r, 0)$ is also saddle.

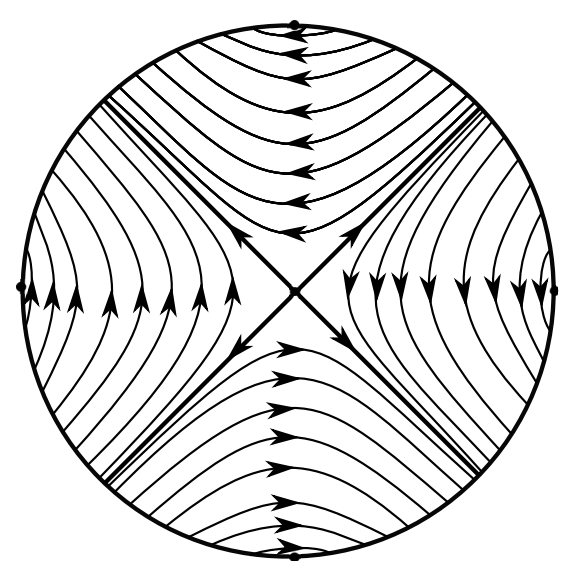

FiguRE 3. Phase portrait in a neighborhood of the equilibrium point $(0, r, 0)$ of system $(2)$.

Lastly system (2) using a convenient time re-parametrization the dynamics of system (2) restricted to the surface $\left\{x^{2}+y^{2}+z^{2}=r, x>\right.$ $0\}$ in a neighborhood of the equilibrium point $(r, 0,0)$ is equivalent to the dynamics of the system

$$
\dot{y}=(\alpha-\gamma) z, \quad \dot{z}=(\beta-\alpha) y .
$$

Since $\alpha<\beta<\gamma$ and system (7) is linear, the equilibrium point $(r, 0,0)$ is a center, and by the symmetry the equilibrium point $(-r, 0,0)$ is also center. This concludes the proof of statement (i).

To show statement (ii) and (iii), denote by $f$ the vector field defined by system (2), and consider the involution $R_{y}(x, y, z)=(x,-y, z)$. Then the vector field $f$ is $R_{y}$-reversible.

We take now the quotient between $\dot{z}$ and $\dot{x}$ in system (6), that is

$$
\frac{d z}{d x}=\frac{(\beta-\alpha) x}{(\gamma-\beta) z} .
$$

Integrating this differential equation we obtain the hyperbolas

$$
(\alpha-\beta) x^{2}+(\gamma-\beta) z^{2}=k,
$$


because $\alpha<\beta<\gamma$. See Figure 3 .

Since $f$ is $R_{y}$-reversible by Lemma 3 the unstable separatrices coming from the saddle $(0,-r, 0)$ until the plane $\{y=0\}$ connect with the stable separatrices of the saddle $(0, r, 0)$. Thus we have two heteroclinic connections.

Again since $f$ is $R_{y}$-reversible, by Lemma 3 each hyperbola of the southern hemisphere connects with a hyperbola of the northern hemisphere, producing a periodic orbit of some of the centers $( \pm r, 0,0)$ and $(0,0, \pm r)$, see Figure 1 . This completes the proof of Theorem 2 .

\section{ACKNOWLEDGMENTS}

This work has been realized thanks to the Brazilian FAPESP grant 2013/24541-0 and Brazilian Capes grant 88881.068462/2014-01.

\section{REFERENCES}

[1] M. Adler And P. van Moerbeke, The algebraic integrability of geodesic flow on $\mathrm{SO}(4)$. Invent. Math. 67(2) (1982), 297-331.

[2] A. T. Fomenko, Integrability and nonintegrability in geometry and mechanics, in Mathematics and its Applications (Soviet Series) 31, Kluwer Academic Publishers Group, Dordrecht, 1988. Translated from the Russian by M. V. Tsaplina.

[3] A. T. Fomenko and V. V. Trofimov, Integrable systems on Lie algebras and symmetric spaces, in Advanced Studies in Contemporary Mathematics 2, Gordon and Breach Science Publishers, New York, 1988. Translated from the Russian by A. Karaulov, P. D. Rayfield and A. Weisman.

[4] J. Llibre, J. Yu, AND X. Zhang, On polynomial integrability of the Euler equations on so(4), J. Geom. Phys. 96 (2015), 36-41.

[5] A. J. Maciejewski, S. I. Popov, And J.-M. Strelcyn, The Euler equations on Lie algebra so(4): an elementary approach to integrability condition, J. Math. Phys.42 (2001), n. 6, 2701-2717.

[6] P. J. Olver, Applications of Lie groups to differential equations, in Graduate Texts in Mathematics 107, Springer-Verlag, New York, 1986.

[7] A. M. Perelomov, Integrable systems of classical mechanics and Lie algebras, Vol. I, Birkhäuser Verlag, Basel, 1990. Translated from the Russian by A. G. Reyman [A. G. Reĭman].

[8] V. V. Trofimov, Introduction to geometry of manifolds with symmetry, in Mathematics and its Applications 270, Kluwer Academic Publishers Group, Dordrecht, 1994. Translated from the 1989 Russian original by G. G. Okuneva.

1 Departamento de Matemática, iBilce - Unesp Universidade Estadual Paulista, Rua Cristovão Colombo 2265, Jardim Nazareth, CEP 15.054-000, Sao José do Rio Preto, SP, Brazil 
2 Departament de Matemàtiques, Universitat Autònoma de Barcelona, 08193 Bellaterra, Barcelona, Spain

3 Instituto de Ciências Naturais Humanas e Sociais, Universidade Federal de Mato Grasso, Avenida Alexandre Ferronato 1.200, 78.557267, Sinop, MT, Brazil 